\title{
Right atrial mass-Multimodality imaging-Massive Lipomatous hypertrophy of the atrial septum
}

\author{
Veera Pavan Kotaru ${ }^{1}$, David Martin², Hemasri Tokala ${ }^{3}$, and Jagadeesh Kalavakunta ${ }^{4}$ \\ ${ }^{1}$ Affiliation not available \\ ${ }^{2}$ Ascension Borgess Hospital \\ ${ }^{3}$ Western Michigan University Homer Stryker M.D. School of Medicine \\ ${ }^{4}$ Michigan State University
}

July 30, 2020

\begin{abstract}
Asymptomatic cardiac neoplasms are often diagnosed incidentally. Right atrial mass close to septum needs careful evaluation and multimodality imaging to manage appropriately. This case highlights the utilization of multimodality imaging and heart team approach in the management of the right atrial mass.
\end{abstract}

\section{Hosted file}

IAS pdf.pdf available at https://authorea.com/users/347493/articles/473155-right-atrialmass-multimodality-imaging-massive-lipomatous-hypertrophy-of-the-atrial-septum 